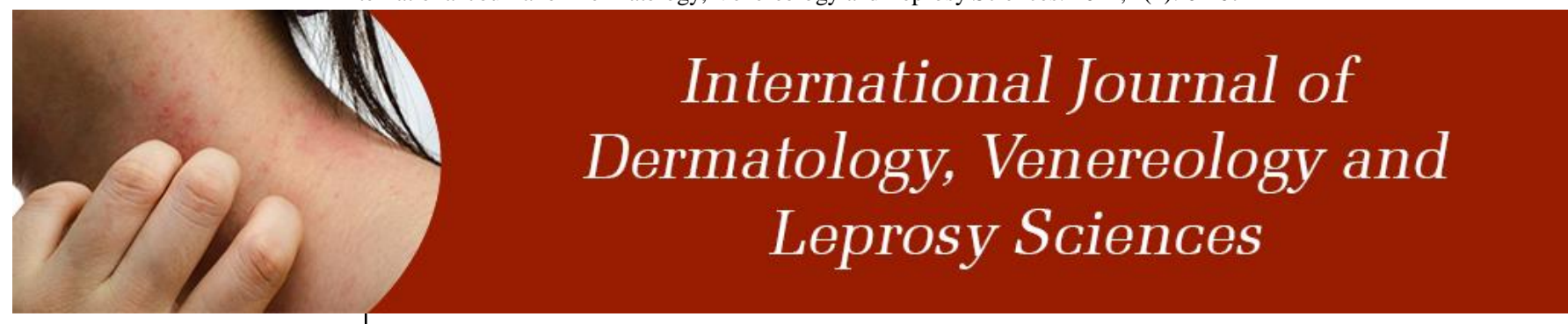

E-ISSN: 2664-942X P-ISSN: 2664-9411 www.dermatologypaper.com/ Derma 2021; 4(1): 01-07 Received: 25-10-2020 Accepted: 03-12-2020

Dr. Suyomi Shah MD, Dermatology Venereology and Leprosy, Dr. DY Patil Medical College, Maharashtra, India

\section{Dr. Krupa Ajmera}

Resident, Dermatology Venereology and Leprosy, Dr. DY Patil Medical College, Maharashtra, India

Dr. Aswathy Radhakrishnan Resident, Dermatology Venereology and Leprosy, Dr. DY Patil Medical College, Maharashtra, India

\section{Dr. Aishwarya Rai} Resident, Dermatology Venereology and Leprosy, Dr. DY Patil Medical College, Maharashtra, India

Dr. Nitin Nadkarni MD, Dermatology Venereology and Leprosy, Dr. DY Patil Medical College, Maharashtra, India

\section{Dr. Sharmila Patil}

Head of Department, Dermatology Venereology and Leprosy, Dr. DY Patil Medical College, Maharashtra, India

Corresponding Author: Dr. Krupa Ajmera Resident, Dermatology Venereology and Leprosy, Dr. DY Patil Medical College, Maharashtra, India

\section{Hair loss in adult females: A clinical and trichoscopic study}

\author{
Suyomi Shah, Krupa Ajmera, Aswathy Radhakrishnan, Aishwarya Rai, \\ Nitin Nadkarni and Sharmila Patil
}

DOI: https://doi.org/10.33545/26649411.2021.v4.i1.53

\begin{abstract}
Hair loss is a matter of concern in any individual irrespective of age and gender, more so in females. This study was undertaken to analyze the clinical patterns of hair loss in women in various disorders based on trichoscopic evaluation. In each patient, hair pull test and trichoscopy was done. Majority of the patients $(67 \%)$ were in the age group of 21-40 years. The most common presentation was hair fall. Hair pull test was positive in $69.2 \%$ cases. The most common diagnosis observed was female pattern hair loss (36.2\%); followed by telogen effluvium, pityriasis sicca, alopecia areata, canities, pediculosis capitis, scalp psoriasis and seborrheic dermatitis. Trichoscopy showed empty hair follicles, focal atrichia, anisotrichosis, increased vellus hair, pilosebaceous units with 1-2 hair, white dots of eccrine ducts, honeycomb pattern. Trichoscopy is an important diagnostic tool especially in patients when diagnosis was unclear. It is an important technique and allowed us to avoid scalp biopsy for difficult cases.
\end{abstract}

Keywords: Hair, Seborrheic dermatitis, Pull test, Trichoscope

\section{Introduction}

Hair loss is a matter of concern in any individual irrespective of age and gender, more so in females. Normal hair cycle results in replacement of every hair on the scalp by 3-5 years. Hair is an essential part of identity for many women. Femininity, sexuality, attractiveness, and personality are symbolically linked to woman's hair. Women are more likely to have lowered quality of life and restrict social contact as compared to men as a result of hair loss [1].

Most hair loss can be broadly classified into THREE types: 1) Non-cicatricial type (potentially reversible) 2) Cicatricial type 3) Due to hair shaft abnormalities.

Women presenting with diffuse hair loss is a very common and challenging problem for dermatologists ${ }^{[2]}$. The condition has several causes and this article discusses the key diagnostic features and management strategies for these three most common causes of nonscarring diffuse hair loss in adult females ${ }^{[3]}$.

Alopecia refers to lack of hair in areas where hair ordinarily would be found. Androgenetic alopecia (AGA), the common baldness is referred so, as there is androgen-mediated conversion of susceptible terminal hair into vellus hair. Female pattern hair loss (FPHL) was originally described as synonymous with androgenetic alopecia ${ }^{[4]}$. FPHL is a non-scarring diffuse alopecia, characterized by a reduction in hair density over the crown and frontal scalp with retention of the frontal hairline. Its prevalence increases with age from approximately $12 \%$ amongst women aged between 20 and 29 years to over $50 \%$ in women over the age of 80 years. A total of 3 patterns have been recognized:

- Diffuse thinning of the crown with preservation of frontal hair line. Ludwig classified it into three grades of severity referred to as Ludwig grades I, II, and III ${ }^{[6]}$.

- Oslen's "Christmas tree pattern" with frontal midline breach and widening of the central part of the scalp without diffuse hair loss ${ }^{[9]}$.

- $\quad$ Thinning associated with bi temporal recession (Hamilton type) ${ }^{[10]}$.

The histological hallmark of FPHL is miniaturization of hair follicles with a progressive transformation of terminal hair follicles into vellus-like follicles. In addition, women with FPHL have more follicles in the telogen phase of hair cycle, and fewer in the anagen phase. 


\section{Prevalence}

Hair loss is common, both in males as well as females, the most prevalent disorders being androgenetic alopecia (male pattern baldness) and alopecia areata, \{both of which are characterized by rapidly cycling miniaturized hair follicles that fail to produce quality terminal hairs. The hair follicle bulbs are not destroyed, so the follicles are able to regenerate and hair regrowth is possible with effective treatment. A About $13 \%$ of premenopausal women and $75 \%$ of women over the age of 65 years, experience some androgenetic alopecia, with a typical presentation of thinning over the crown. Alopecia areata is more commonly associated with acquired thyroid disease, diabetes and collagen diseases, though it is often associated with stress. It affects $0.1 \%-0.2 \%$ of people with a lifetime risk of $1.7 \%$.

FPHL is more frequent than previously thought; the incidence of PHL in women may equal men. FPHL may begin at any age following puberty and the prevalence increases in post-menopausal age with a possible hormonal influence. In general, FPHL has its onset during the reproductive years. More severe cases of the disease are already noticeable at puberty. However, there is a greater demand for treatment among patients aged 25-40 years. There is a second peak incidence at menopause, between 50 and 60 years of age. FPHL, is associated with hyperandrogenic conditions, like polycystic ovarian syndrome (PCOS), although most patients may not display any signs of the same.
Women with Metabolic Syndrome had higher prevalence for FPHL than others $(\mathrm{OR}=1.7)$. This relationship was, however, not observed in male population, suggesting that follicle miniaturization in FPHL and MPA arise from different stimuli.

\section{Materials and Methods}

A total of 650 patients between duration of 2 years were included in the study conducted in the department of Dermatology, Venereology and Leprosy, DY Patil Hospital, Nerul Navi Mumbai. Inclusion criteria were adult female above age of 18 years presenting to our OPD with hair loss. All were informed regarding the study and their consent was obtained. Demographic data such as name, age, gender etc. was recorded. Detailed history was recorded and clinical photograph were taken. In each patient hair pull test and trichoscopy was done. Findings obtained were documented in a specially prepared proforma and photography. The quantitative data was represented as their mean \pm SD. Categorical and nominal data was expressed in percentage. All analysis was carried out by using SPSS software version 21. P value less than 0.05 was considered significant.

\section{Results}

Maximum of the patients $(67 \%)$ were in the age group of 21-40 years with mean age being $34.17+/-9.42$ years.

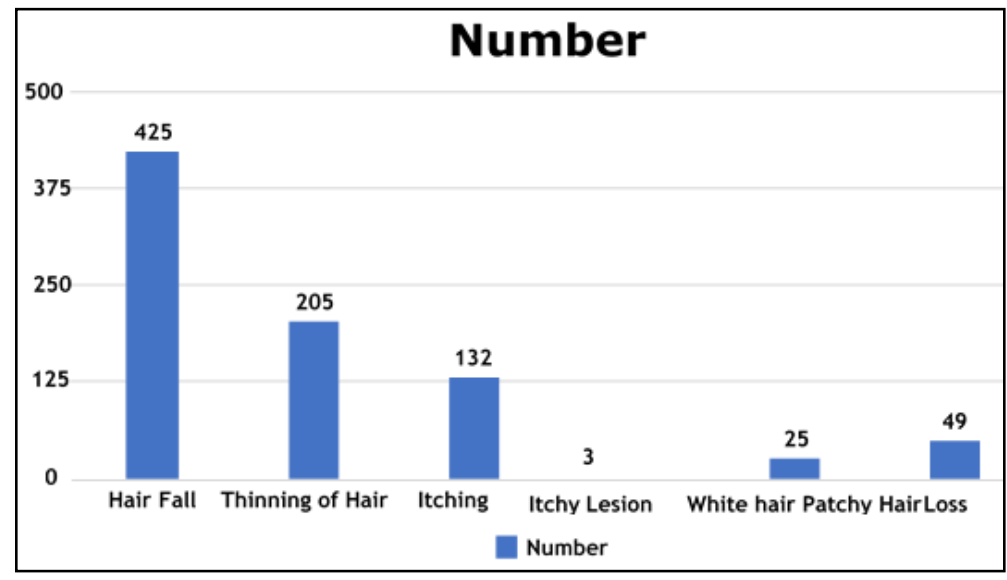

Graph 1: Distribution of study subjects as per symptoms

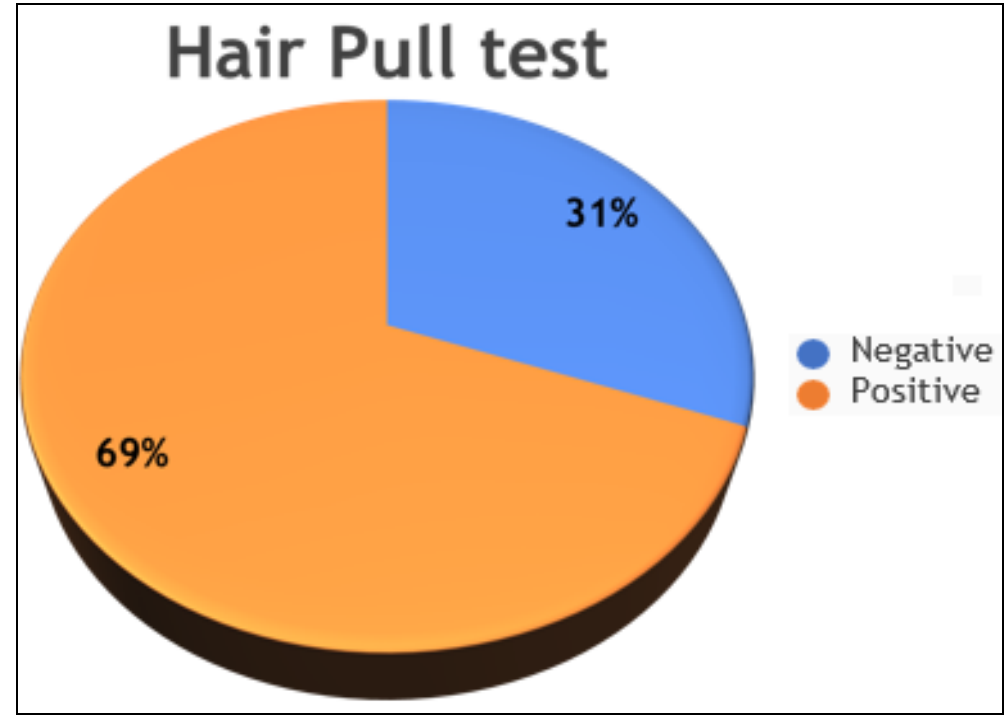

Graph 2: Distribution of study subjects as per hair pull test 
Graph II shows that hair pull test was positive in $69.2 \%$ cases Most common diagnosis as observed in present study was also FPHL (female pattern hair loss), which was observed in $36.2 \%$ of the patients. Second common diagnosis was telogen effluvium $(30.2 \%)$ followed by pityriasis sicca $(10.9 \%)$, alopecia areata $(5.8 \%)$, canities (3.8\%) pediculosis capitis $(3.5 \%)$, scalp psoriasis $(2.8 \%)$ and seborrheic dermatitis $(1.8 \%)$.
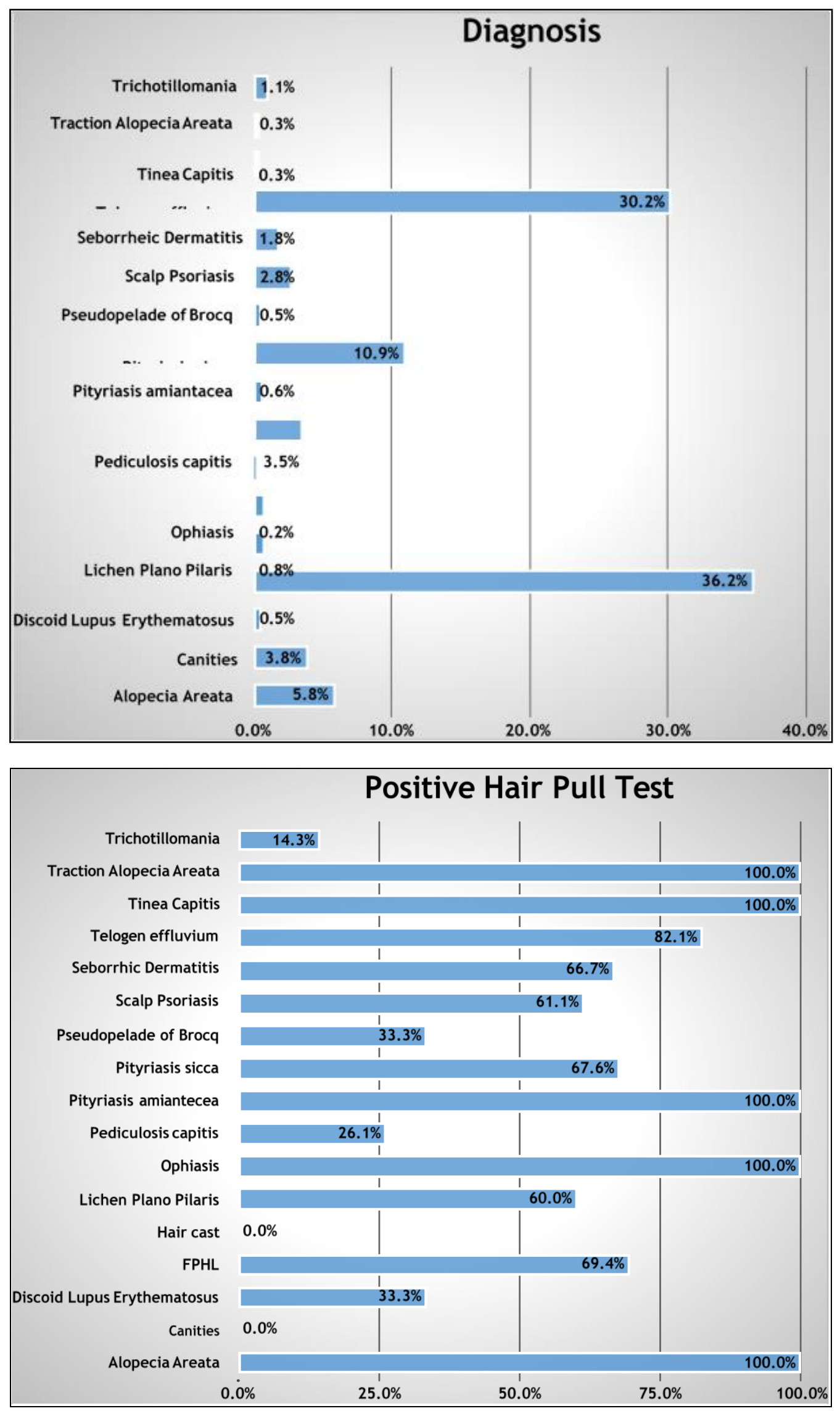


\section{Alopecia areata}

Present study observed increased vellus hair in $65.8 \%$ of the patients, broken hair in $50 \%$ of the patients, exclamation mark hair in $42.1 \%$, yellow dots in $31.6 \%$, pigtail sign was observed in $15.8 \%$ and black dots in $13.2 \%$. Trichoscopic finding in various diseases are as follows

\section{Canities}

Mutliple depigmented white hair seen was seen in all cases of canities.

\section{Discoid lupus erythematosus}

Present study observed loss of hair follicles in $100 \%$ of the patients, perifollicular erythema in $66.7 \%$, amorphous white and brown areas and perifollicular hyperpigmentation in $33.3 \%$ of the patients.

\section{Female pattern hair loss}

Trichoscopy findings of the present study revealed anisotrichosis in $97.9 \%$ of the patients of FPHL while pilosebaceous units with $1 / 2$ terminal hair was observed in $78.3 \%$ of the patients of the present study.

Other findings observed were empty hair follicles in $71.9 \%$, brown peripilar halo in $62 \%$, increased vellus hair in $54 \%$ and honey comb pattern in $14.5 \%$ of the patients.

\section{Telogen effluvium}

Present study observed honey comb pattern in $68.4 \%$ of the Telogen effluvium patients, decreased hair density in $50 \%$ of the patients and empty hair follicles were observed in $49 \%$ of the patients.

\section{Hair cast}

White tubular structures were seen in all cases of hair Cast.

\section{Lichen Plano Pilaris}

Present study observed honey comb pattern in $80 \%$ of the patients of LPP, blue grey dots were seen in $60 \%$ of the patients, other features observed were amorphous white and brown area, peripilar casts, follicular plugging, and arborizing red loops in about $20 \%$ of the patients.

\section{Ophiasis}

In a solitary case of Ophiasis, trichoscopy observed increase vellus hair and pig tail sign.

\section{Pediculosis Capitis}

On trichoscopy, nits were observed in all cases of Pediculosis Capitis.

\section{Pityriasis amiantacea}

On trichoscopy, hyperkeratotic scales were observed in all cases of Pityriasis amiantacea.

\section{Pityriasis Sicca}

On trichoscopy, white adherent scales were observed in all cases of Pityriasis sicca.

\section{Pseudopelade of Brocq}

In 3 cases of Pseudopelade of Brocq, trichoscopy observed loss of hair follicles and porcelain white areas in all 3 cases.

\section{Scalp Psoriasis}

On trichoscopy, all cases of scalp psoriasis showed white adherent scales while signet ring vessels and hidden hair were seen in $66.7 \%$ and $44.4 \%$ cases. Red dots and globules were seen in one third of the cases.

\section{Seborrheic Dermatitis}

Yellowish greasy scales were seen in all cases of seborrheic dermatitis while black dots and brown dots were seen in $33.3 \%$ and $16.7 \%$ cases respectively.

\section{Tinea capitis}

$\mathrm{KOH}$ - fungal hyphae and spores seen in both cases of tinea capitis.

\section{Tractional alopecia}

Our study observed broken hairs in all patients with tractional AA and increased vellus hair and white dots in half of the patients.

\section{Trichotillomania}

Broken hair were seen in all cases of trichotillomania while black dots, V-shaped hair and follicular hemorrhage were seen in $57.1 \%, 28.6 \%$ and $14.3 \%$ cases respectively.

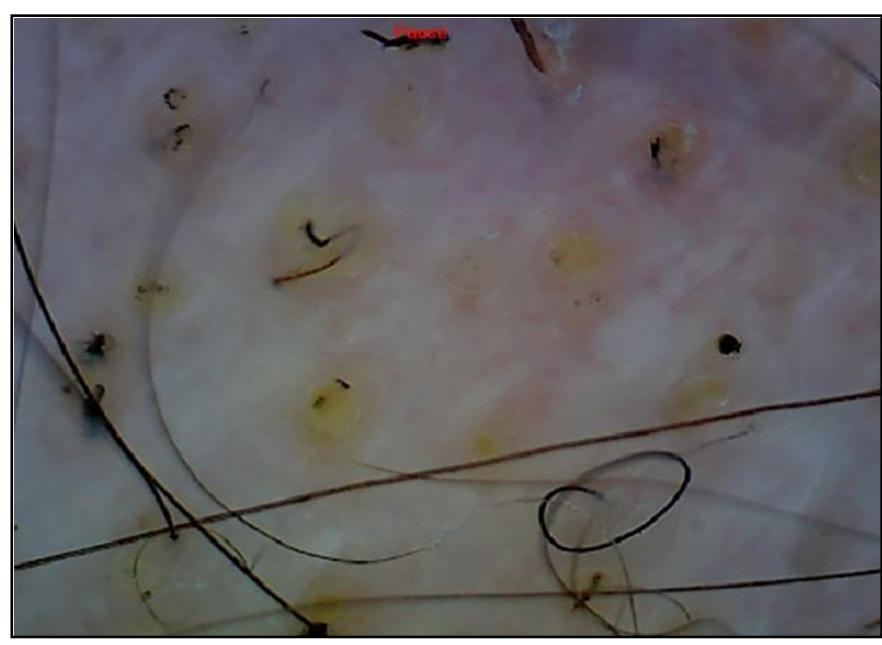

Fig 1: Alopecia Areata

Trichoscopy showing yellow dots, pigtail sign, broken hair, black dots.

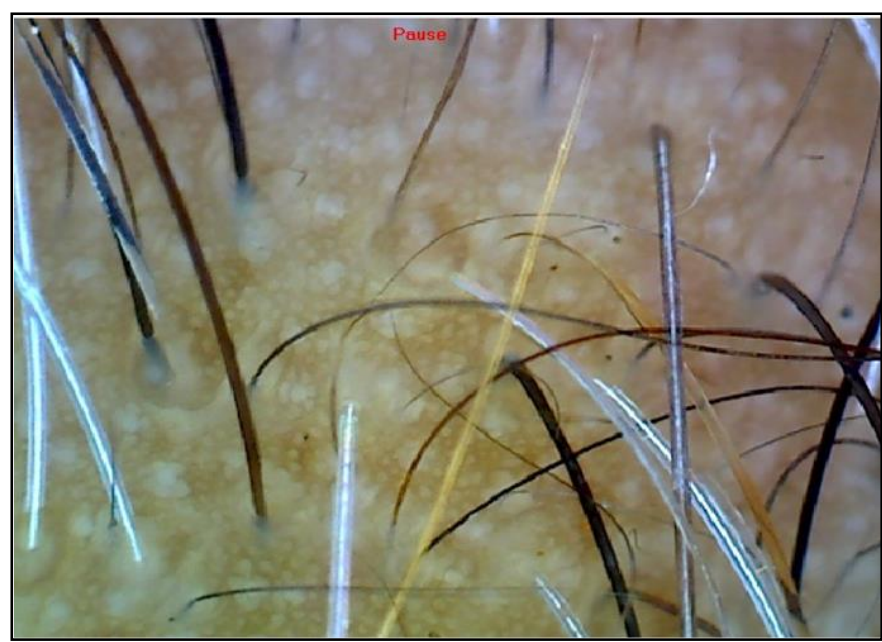

Fig 2: Canitis

Trichoscopy showing various depigmented hair. 


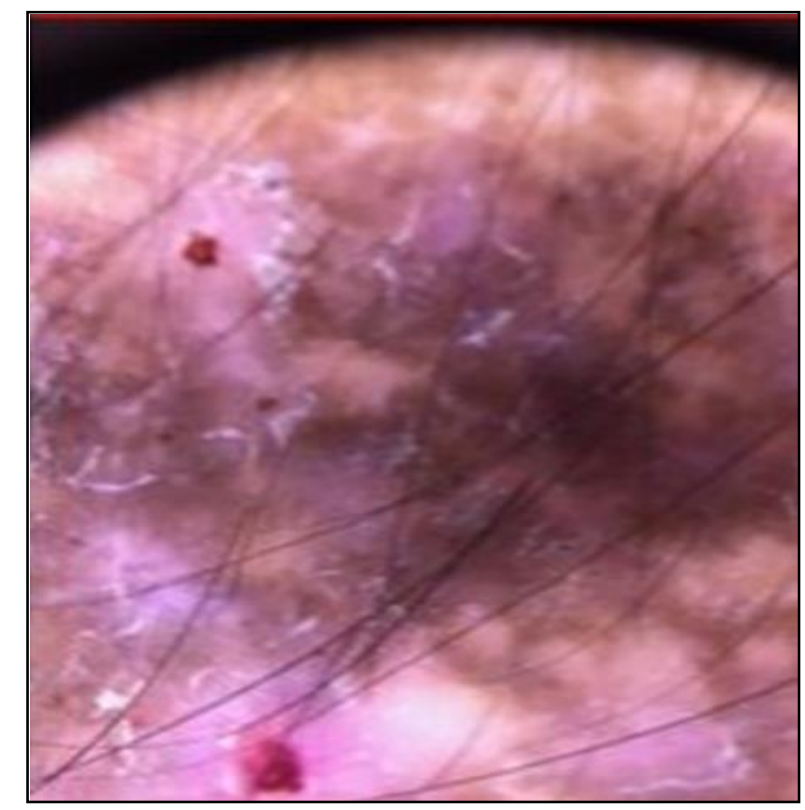

Fi g 3: Discoid Lupus Erythematosus

Trichoscopy showing loss of hair follicles, amorphous white and brown areas, perifollicular erythema, perifollicular hyperpigmentation along with scales.

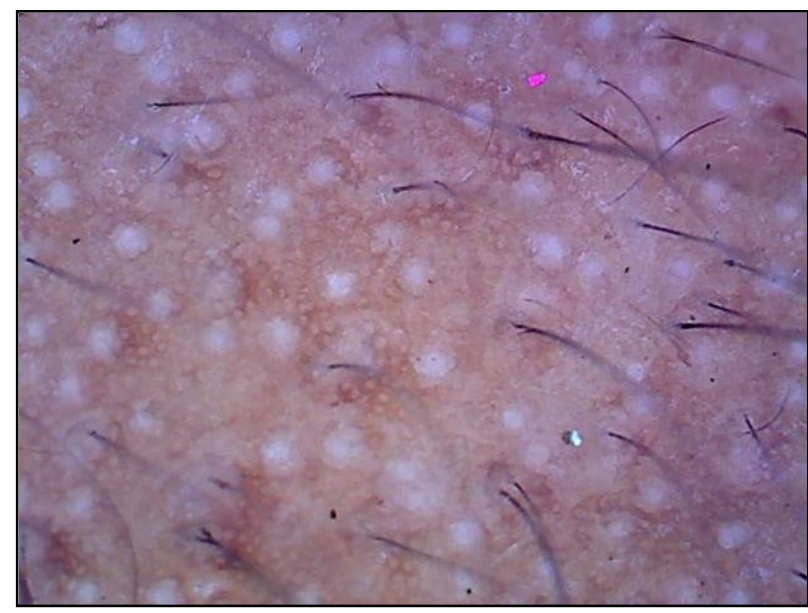

Fig 4: Female Patterned Hair Loss

Trichoscopy showing empty hair follicles, focal atrichia, anisotrichosis, increased amount of vellus hair, pilosebaceous units with 1-2 hair, white dots of eccrine ducts, honeycomb pattern.

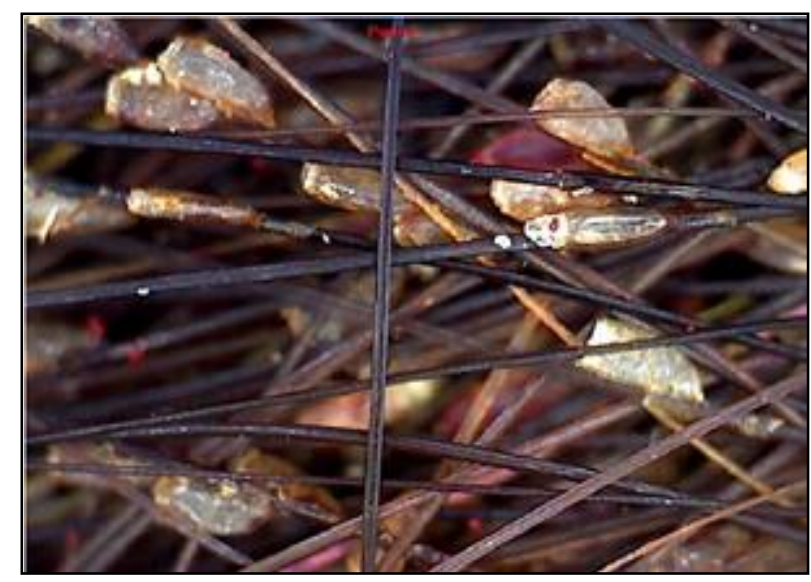

Fig 5: Pediculosis capitis.
Trichoscopy showing nits attached to the hair shafts.

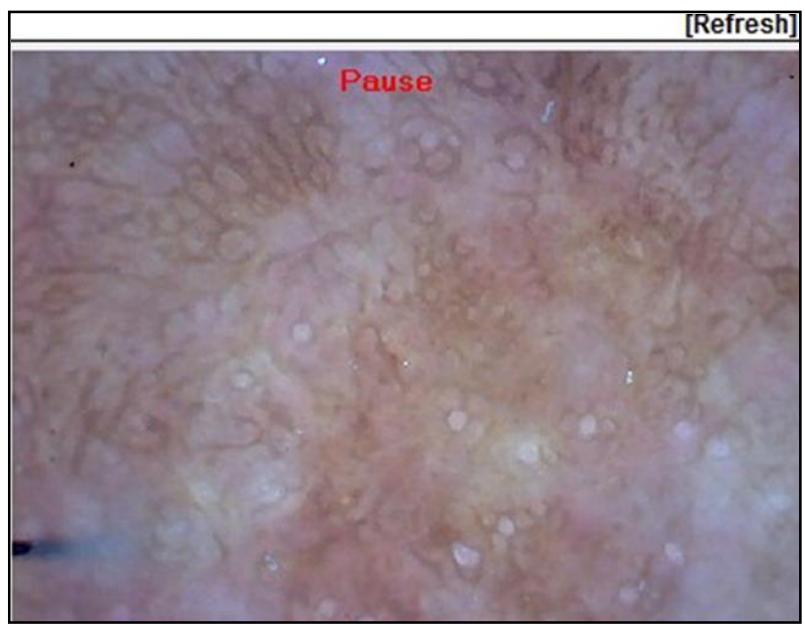

Fig 6: Pseudopelade of Brocq

Trichoscopy showing loss of hair follicles along with porcelain white areas.

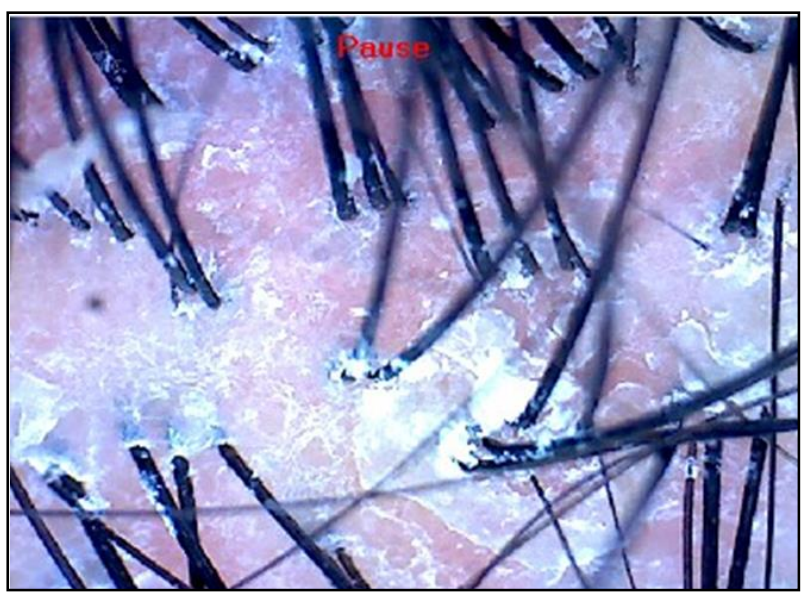

Fig 7: Scalp Psoriasis

Trichoscopy showing white adherent scales on an erythematous background and hidden hair.

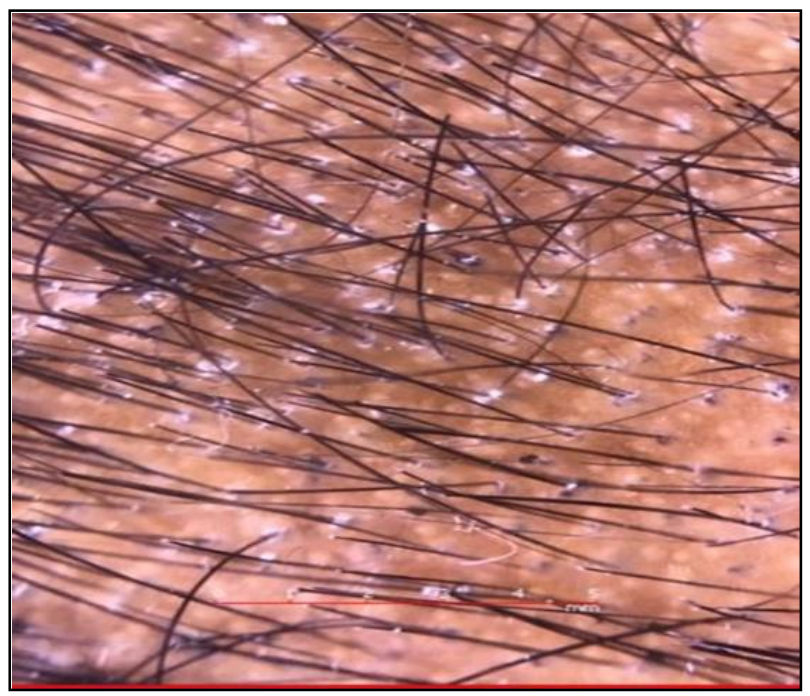

Fig 8: Tinea Capitis

Trichoscopy showing broken hair, black dots, peripilar sign, comma shaped hair. 


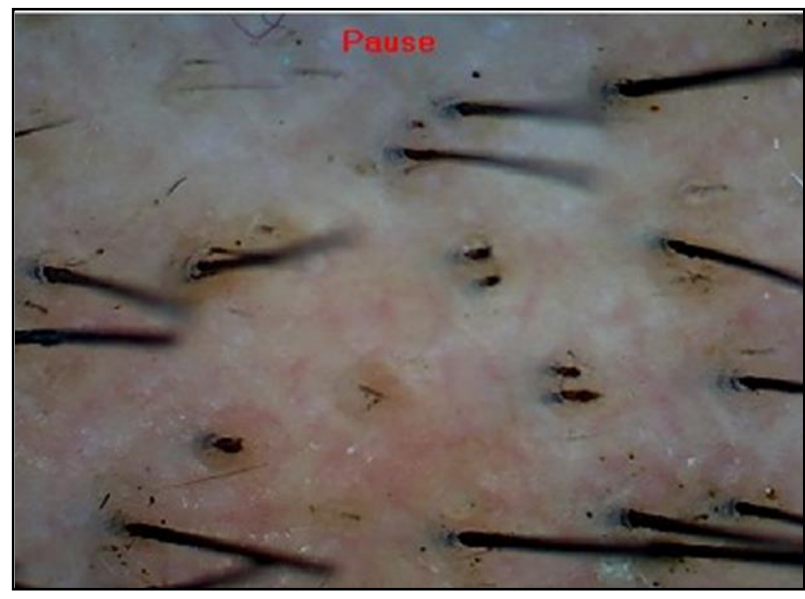

Fig 9: Trichotillomania

Trichoscopy showing v shaped hair, broken hair.

\section{Discussion}

Hair loss is a matter of concern in any individual irrespective of age and gender, more so in females. Psychiatric disorders are more prevalent in patients with alopecia than in general population, suggesting that those with alopecia may be at higher risk for developing a serious depressive episodes, anxiety disorders, social phobia, or paranoid disorder ${ }^{[6]}$.

Chronic hair loss frequently affects female patients, but there is little or no objective technology available to aid the dermatologist in setting a proper diagnosis and in monitoring treatment efficacy ${ }^{[7]}$. In particular, it may be difficult to differentiate between female androgenic alopecia (FAGA), a subtype of female pattern hair loss, and other pathologies ${ }^{[8]}$.

Recently, trichoscopy (scalp dermoscopy), being a hot-spot in the field of trichology has led to the establishment of diagnostic features of some hair disorders like alopecia areata, trichotillomania, tinea capitis among others. The need for non-invasive, but precise tool for effluvium and other pathologies diagnosis led us implement trichoscopy for diagnostic purposes in hair loss. However, literature investigating features of FPHL in females are few.9 Present study was undertaken to study the clinical patterns of hair loss which manifest in various disorders and to establish appropriate and early diagnosis and correlate the pattern of hair loss with the diagnosis with the help of a trichoscope.

The present study was conducted on 650 female patients who presented with the hair loss diseases and pattern of hair loss was studied with the help of a trichoscope. Maximum of the patients $(67 \%)$ were in the age group of $21-40$ years with mean age being $34.17+/-9.42$ years. Sillani C et al. ${ }^{[10]}$, the mean age was $34.9 \pm 11.1$ which is also in accordance with the age of the patients in the present study.

We found that the most common symptom of presentation was hair fall in $65.4 \%$, thinning of hair was reported by $31.5 \%$ which is also a direct indication of hair fall and $20.3 \%$ of the patients presented with complaints of itching. Various other studies have also reported patients presenting with similar complaints. Ludwig $\mathrm{E}$ has mentioned that the majority of patients complained of reduced size of plait, prior to the onset of the hair thinning was noted in a small percentage of patients which is exactly observed by present study.

We found that hair pull test was positive in $69.2 \%$ cases.
The most common diagnosis as observed in present study was also FPHL (female pattern hair loss), which was observed in $36.2 \%$ of the patients. Second common diagnosis was telogen effluvium (30.2\%) followed by pityriasis sicca $(10.9 \%)$, alopecia areata $(5.8 \%)$, canities (3.8\%) pediculosis capitis $(3.5 \%)$, scalp psoriasis $(2.8 \%)$ and seborrheic dermatitis (1.8\%). However, hair cast, lichen plano pilaris, ophiasis, discoid lupus erythematosus, pityriasis amiantacea, pseudopelade of Brocq, tinea capitis, traction alopecia areata and trichotillomania.

Herskovitz I et al. ${ }^{[1]]}$ they reported that the most important diseases to consider after FPHL includes Chronic Telogen Effluvium (CTE), Permanent Alopecia after Chemotherapy (PAC), Alopecia Areata Incognito (AAI) and Frontal Fibrosing Alopecia (FFA). Another study by Ravikiran SP et al. ${ }^{[12]}$ involving the cohort of FPHL among the patients actively seeking treatment for their hair loss, they found that the FPHL accounts for $15.3 \%$ of diffuse hair loss in women. Pull test is an invasive diagnostic technique, very easy to perform and to repeat. Pull test is very helpful to rapidly determine the ongoing activity and severity of any kind of hair loss. Hair pull test was positive in $65.4 \%$ of the patients in present study, giving $100 \%$ positivity for Pityriasis amiantacea, Alopecia Areata, ophiasis, tinea capitis and traction alopecia areata, $82 \%$ positivity in Telogen effluvium and $69.4 \%$ positivity in FLPH cases and $67.6 \%$ in pityriasis sicca, $66.7 \%$ in seborrheic dermatitis.

\section{Conclusion}

Trichoscopy is a useful diagnostic tool in differentiating non-scarring from scarring alopecia and alopecia areata from other patchy alopecias, as well as in diagnosing early androgenetic alopecia and tinea capitis. In our study, trichoscopy helped to reach a conclusive diagnosis in patients in whom the diagnosis was not clear. Trichoscopy is an important technique and allowed us to avoid scalp biopsy for difficult cases.

Authors found that the most common diagnosis as observed in present study was FPHL (female pattern hair loss), which was observed in $36.2 \%$ of the patients. Second common diagnosis was telogen effluvium followed by pityriasis sicca, alopecia areata, canities, pediculosis capitis, scalp psoriasis and seborrheic dermatitis.

\section{References}

1. Yi SM, Son SW, Lee KG, Kim SH, Lee SK, Cho ER, et al. Gender-specific association of androgenetic alopecia with metabolic syndrome in a middle-aged Korean population. Br J Dermatol 2012;167:306-13.

2. Abdel Fattah NS, Darwish YW. Androgenetic alopecia and insulin resistance: are they truly associated? Int $\mathbf{J}$ Dermatol 2011;50:417-22.

3. Mansouri P, Mortazavi M, Eslami M, Mazinani M. Androgenetic alopecia and coronary artery disease in women. Dermatol Online J 2005;11:2.

4. Arias-Santiago S, Gutiérrez-Salmerón MT, CastelloteCaballero L, Buendía-Eisman A, Naranjo-Sintes R. Androgenetic alopecia and cardiovascular risk factors in men and women: a comparative study. J Am Acad Dermatol 2010;63:420-9.

5. Ross EK, Tan E, Shapiro J. Update on primary cicatriciai alopecias. J Am Acad Dermatoi 2005;53:137.

6. Somani N, Bergfeld WF. Cicatriciai alopecia: 
classification and histopathol- ogy. Dermatoi Ther 2008;21:221-37.

7. Lopez-Tintos BO, Gareia-Hidalgo L, Orozco-Topete R. Dermoscopy in active discoid lupus. Arch Dermatoi 2009; $145: 358$.

8. El Sayed MH, Abdallah MA, Aly DG, Khater NH. Association of metabolic syndrome with female pattern hair loss in women: A case-control study. Int J Dermatol 2016;55:1131-7.

9. Ramos PM, Miot HA. Female pattern hair loss: A clinical and pathophysiological re- view. An Bras Dermatol 2015;90(4):529-43.

10. McDonald KA, Shelley AJ, Colantonio S, Beecker J. Hair pull test: Evidence-based up- date and revision of guidelines. J Am Acad Dermatol 2017;76:472-7.

11. Herskovitz I, Tosti A. Female pattern hair loss. Int J Endocrinol Metab 2013;11(4):9860.

12. 12.Ravikiran SP, Syrti C, Shashidhar T. An epidemiological study of female pattern hair loss at a referral centre in South India. Indian Journal of Clinical and Experimental Dermatology 2016;2(3):106-10. 